Supplement of Hydrol. Earth Syst. Sci., 22, 1437-1452, 2018

https://doi.org/10.5194/hess-22-1437-2018-supplement

(C) Author(s) 2018. This work is distributed under

the Creative Commons Attribution 3.0 License.

(c) (1)

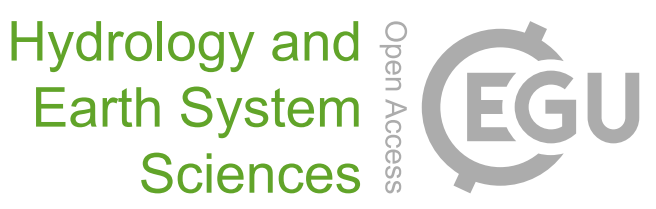

Supplement of

\title{
Testing and development of transfer functions for weighing precipitation gauges in WMO-SPICE
}

John Kochendorfer et al.

Correspondence to: John Kochendorfer (john.kochendorfer@noaa.gov)

The copyright of individual parts of the supplement might differ from the CC BY 3.0 License. 


\section{Transfer functions}

Transfer function coefficients for the transfer functions that were developed, tested, and recommended are listed in Tables 1

- 4. The equations used in the tables are included here for ease of reference:

$C E=e^{-a(U)\left(1-\tan ^{-1}\left(b\left(T_{a i r}\right)\right)+c\right)}$

5 where $C E$ is the catch efficiency, $U$ is the mean wind speed, $T_{\text {air }}$ is the mean air temperature, and $a, b$, and $c$ are coefficients fit to the data. Equation 2 was also fit to the precipitation measurements:

$C E=(a) e^{-b(U)}+c$

where $a, b$, and $c$ are coefficients fit to the data. Equation 1 was fit as a function of wind speed and air temperature, while Equation 2 was fit separately to solid and mixed precipitation measurements as a function of wind speed only. In the latter

10 case, precipitation type was determined using air temperature, with solid precipitation defined as $T_{\text {air }}<-2{ }^{\circ} \mathrm{C}$, and mixed defined as $2{ }^{\circ} \mathrm{C} \geq T_{\text {air }} \geq-2{ }^{\circ} \mathrm{C}$. For some of the gauges examined here, Eq. 2 unrealistically over-predicted catch efficiency at low wind speeds when insufficiently constrained by the available measurements, and in these cases a more constrained function was used to describe realistic corrections:

$$
C E=(a) e^{-b(U)}+(1-a)
$$

15 Tables

\begin{tabular}{c|cc|cc|ccc|c} 
Wind Speed & \multicolumn{2}{|c|}{ Eq. 3, $\boldsymbol{f}(\boldsymbol{U}$, mixd $)$} & \multicolumn{2}{|c|}{ Eq. 3, $\boldsymbol{f}(\boldsymbol{U}$, solid $)$} & \multicolumn{2}{|c|}{ Eq. 1 $\boldsymbol{f}\left(\boldsymbol{U}, \boldsymbol{T}_{\text {air }}\right)$} & $\boldsymbol{U}_{\text {thresh }}$ \\
& $\boldsymbol{a}$ & $\boldsymbol{b}$ & $\boldsymbol{a}$ & $\boldsymbol{b}$ & $\boldsymbol{a}$ & $\boldsymbol{b}$ & $\boldsymbol{c}$ & (m s $\left.^{-1}\right)$ \\
\hline$f\left(U_{G H}\right)$ & 23.3 & 0.00227 & 4.00 & 0.0334 & 0.0633 & 0.67 & 0.26 & 6.1 \\
$f\left(U_{10 m}\right)$ & 19.7 & 0.00196 & 4.53 & 0.0215 & 0.0465 & 0.66 & 0.25 & 8
\end{tabular}

Table S1. Shielded MRW500 transfer function coefficients for gauge-height (GH) and $10 \mathrm{~m}$ wind speeds. Coefficients for Eq. 3 and Eq. 1 are provided, along with the maximum wind speed threshold $\left(U_{t h r e s h}\right)$, above which the transfer function should be applied by forcing the wind speed down to $U_{\text {thresh }}$.

\begin{tabular}{c|ccc|ccc|ccc|c} 
Wind Speed & \multicolumn{3}{|c|}{ Eq. 2, $\boldsymbol{f}(\boldsymbol{U}$, mixd $)$} & \multicolumn{3}{|c|}{ Eq. 2, $\boldsymbol{f}(\boldsymbol{U}$, solid $)$} & \multicolumn{3}{|c|}{ Eq. 1 $\boldsymbol{f}\left(\boldsymbol{U}, \boldsymbol{T}_{\text {air }}\right)$} & \multicolumn{2}{c}{$\boldsymbol{U}_{\text {thresh }}$} \\
& $\boldsymbol{a}$ & $\boldsymbol{b}$ & $\boldsymbol{c}$ & $\boldsymbol{a}$ & $\boldsymbol{b}$ & $\boldsymbol{c}$ & $\boldsymbol{a}$ & $\boldsymbol{b}$ & $\boldsymbol{c}$ & $\left(\mathbf{m ~ s}^{-\mathbf{1}}\right)$ \\
\hline$f\left(U_{G H}\right)$ & 0.982 & 0.0201 & 0.00 & 0.829 & 0.213 & 0.32 & 0.0404 & 0.4247 & 0.00 & 6.1 \\
$f\left(U_{10 m}\right)$ & 0.98 & 0.0137 & 0.00 & 0.886 & 0.143 & 0.25 & 0.0312 & 0.427 & 0.00 & 8
\end{tabular}

20 Table S2. Double-Alter transfer function coefficients for gauge-height (GH) and 10 m wind speeds. Coefficients for Eq. 2 and Eq. 1 are provided, along with the maximum wind speed threshold $\left(U_{\text {thresh }}\right)$, above which the transfer function should be applied by forcing the wind speed down to $U_{\text {thresh }}$. Also note that the Eq. 1 form of this function should only be used for $T_{a i r}$ is $\leq 5{ }^{\circ} \mathrm{C}$. For $T_{a i r}$ is $>5{ }^{\circ} \mathrm{C}$, use the Eq. 1 type coefficients from K2017b. 


\begin{tabular}{|c|c|c|c|c|c|c|c|c|c|c|}
\hline \multirow[t]{2}{*}{ Wind Speed } & \multicolumn{3}{|c|}{ Eq. $2, f(U$, mixd $)$} & \multicolumn{3}{|c|}{ Eq. $2, f(U$, solid $)$} & \multicolumn{3}{|c|}{ Eq. $1 f\left(U, T_{a i r}\right)$} & \multirow{2}{*}{$\begin{array}{l}U_{\text {thresh }} \\
\left(\mathrm{m} \mathrm{s}^{-1}\right)\end{array}$} \\
\hline & $a$ & $\boldsymbol{b}$ & $c$ & $a$ & $b$ & $c$ & $a$ & $b$ & $c$ & \\
\hline$f\left(U_{G H}\right)$ & 0.260 & 1.512 & 0.95 & 1.068 & 0.049 & 0.00 & 0.0146 & 0.27 & 0.05 & 6.1 \\
\hline$f\left(U_{10 m}\right)$ & 0.254 & 1.052 & 0.95 & 1.075 & 0.039 & 0.00 & 0.0110 & 0.29 & 0.08 & 8 \\
\hline
\end{tabular}

Table S3. Belfort double-Alter transfer function coefficients for gauge-height (GH) and 10 m wind speeds. Coefficients for Eq. 2 and Eq. 1 are provided, along with the maximum wind speed threshold $\left(U_{\text {thresh }}\right)$, above which the transfer function should be applied by forcing the wind speed down to $U_{\text {thresh }}$.

5

\begin{tabular}{l|ccc|ccc|c} 
Wind Speed & \multicolumn{3}{|c|}{ Eq. 2, $\boldsymbol{f}(\boldsymbol{U}$, mixd $)$} & \multicolumn{3}{|c|}{ Eq. 2, $\boldsymbol{f}(\boldsymbol{U}$, solid $)$} & $\boldsymbol{U}_{\text {thresh }}$ \\
& $\boldsymbol{a}$ & $\boldsymbol{b}$ & $\boldsymbol{c}$ & $\boldsymbol{a}$ & $\boldsymbol{b}$ & $\boldsymbol{c}$ & $\left(\mathbf{m ~ s}^{-\mathbf{1}}\right)$ \\
\hline$f\left(U_{G H}\right)$ & 0.492 & 0.00 & 0.492 & 0.187 & 0.410 & 0.875 & 6.1 \\
$f\left(U_{\text {IOm }}\right)$ & 0.492 & 0.00 & 0.492 & 0.186 & 0.230 & 0.875 & 8
\end{tabular}

Table S4. Small DFIR (SDFIR) transfer function coefficients for gauge-height (GH) and $10 \mathrm{~m}$ wind speeds. Coefficients for Eq. 2 are provided, along with the maximum wind speed threshold $\left(U_{\text {thresh }}\right)$, above which the transfer function should be applied by forcing the wind speed down to $U_{\text {thresh }}$. The recommended Eq. 1 type SDFIR transfer function is available in K2017b. 